\title{
Who are we?
}

$\mathrm{BC}$

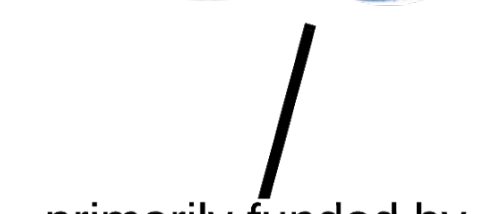

primarily funded by

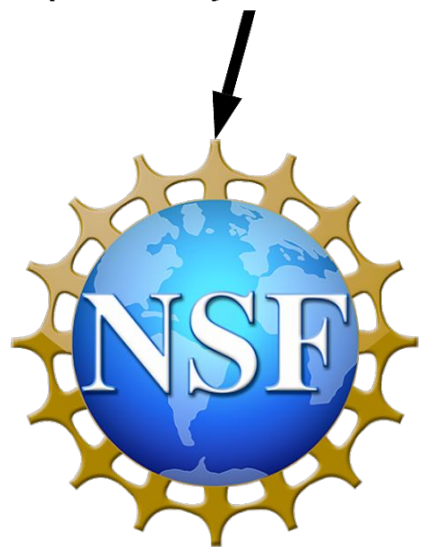

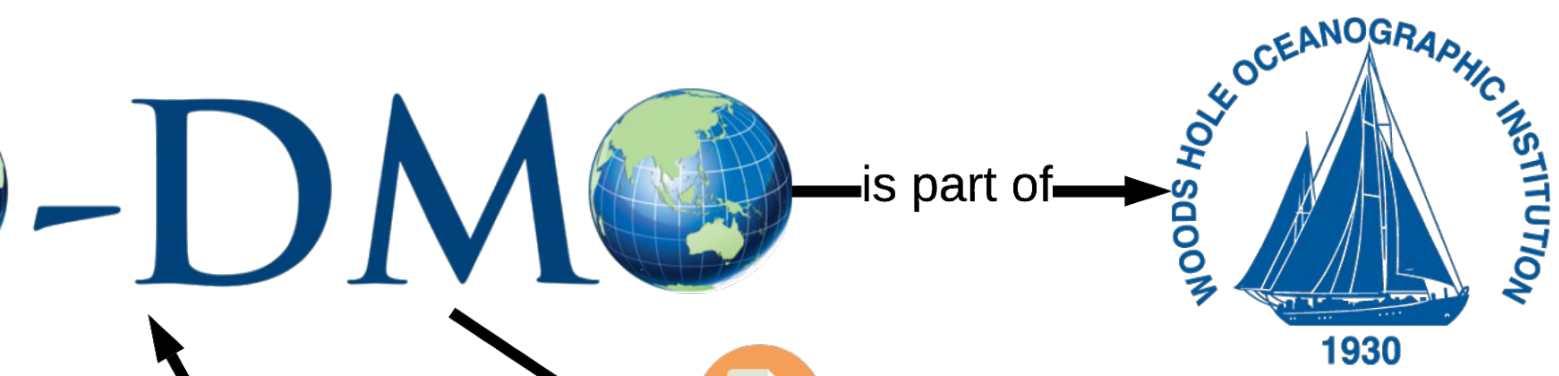

provides data access

$>$ submit data

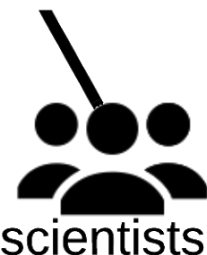




\section{What researchers do we work with?}

National Science Foundation

$>$ Biological Oceanography

$>$ Chemical Oceanography

$>$ Division of Polar Programs Antarctic

Organisms and Ecosystems program.
Gordon and Betty Moore

$>$ Marine Microbiology Initiative

Ad-hoc, fee-based support

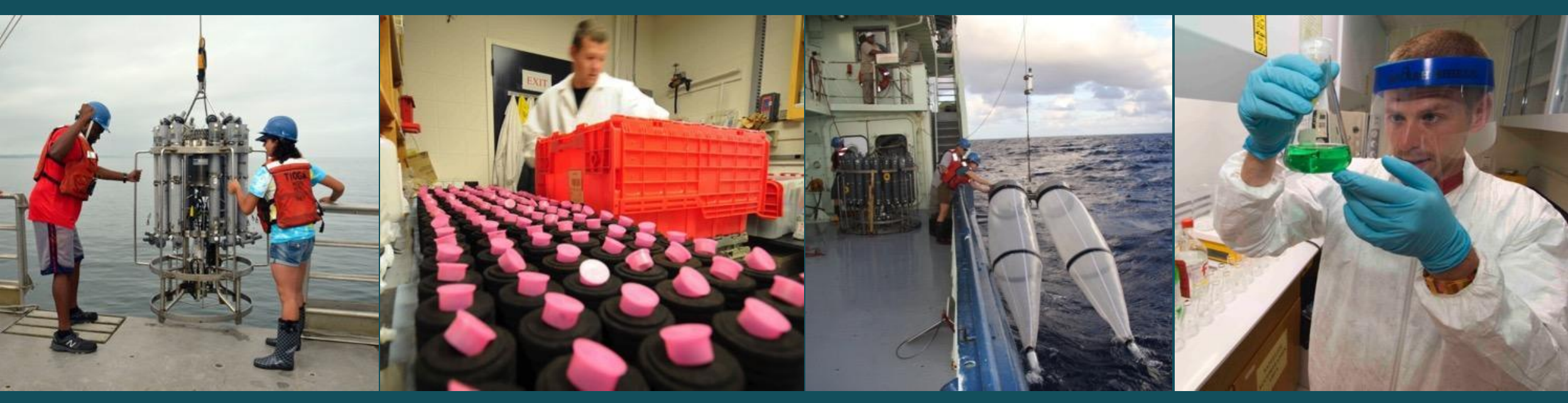




\section{Data Types at BCO-DMO}

Abundance and Biomass

Algal Metabolites

Acoustic Backscatter

Carbonate System Parameters

Current Velocity

Chlorophyll and Accessory Pigments

Dissolved and Particulate Nutrients

Optical Properties

'Omics-related

Mixed Layer Depth

Primary Production

Potential Density

Taxon Group/Species

Trace Element Isotopes

Sedimentation Rate

Sound Velocity

Snow/Ice Cover and Thickness

Spectral Counts

Turbidity

Temperature, Salinity, Oxygen

Trap Type

Wave Height

Wind Direction and Speed Zenith Angle 


\section{Data submission process}

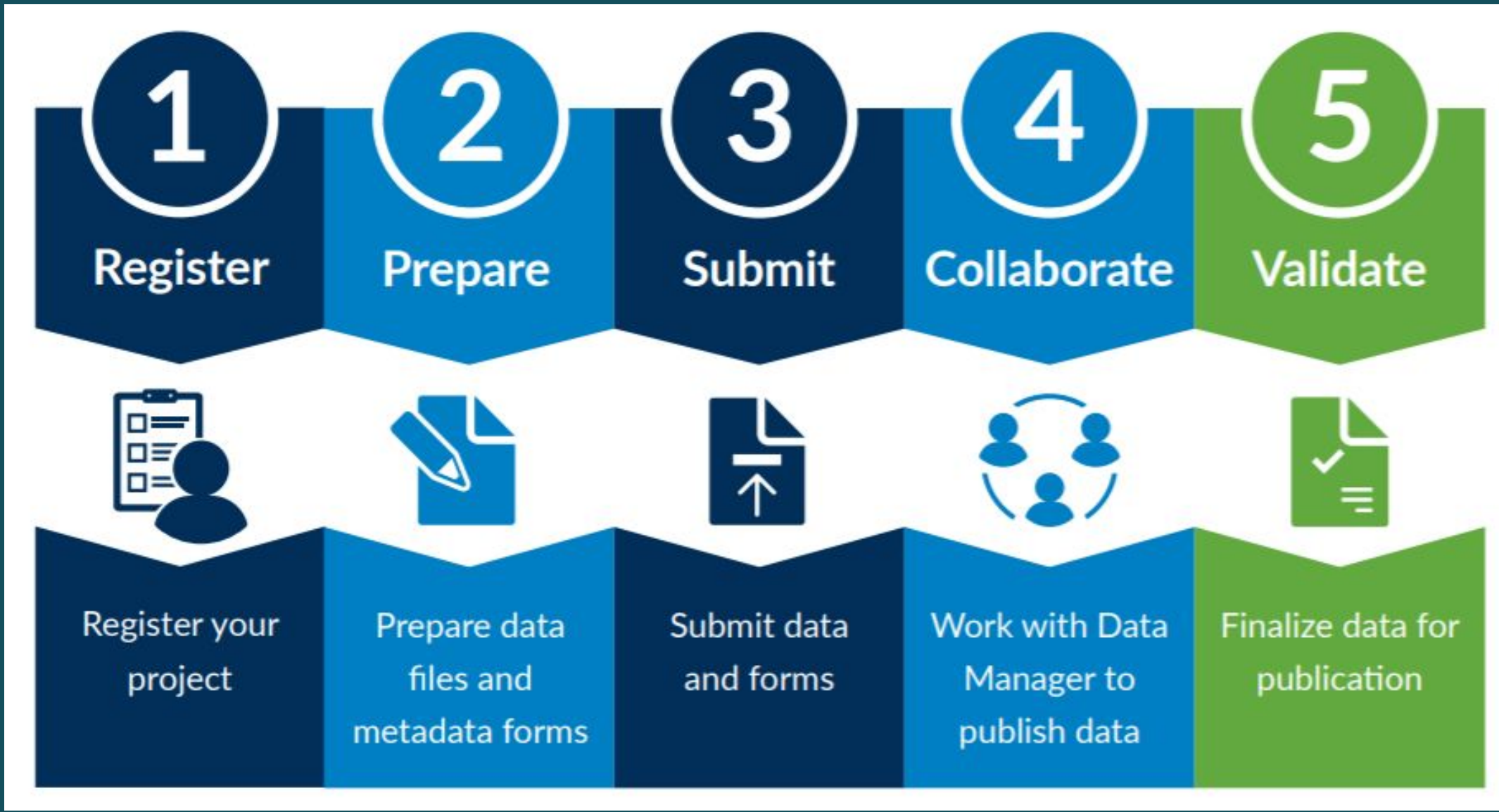



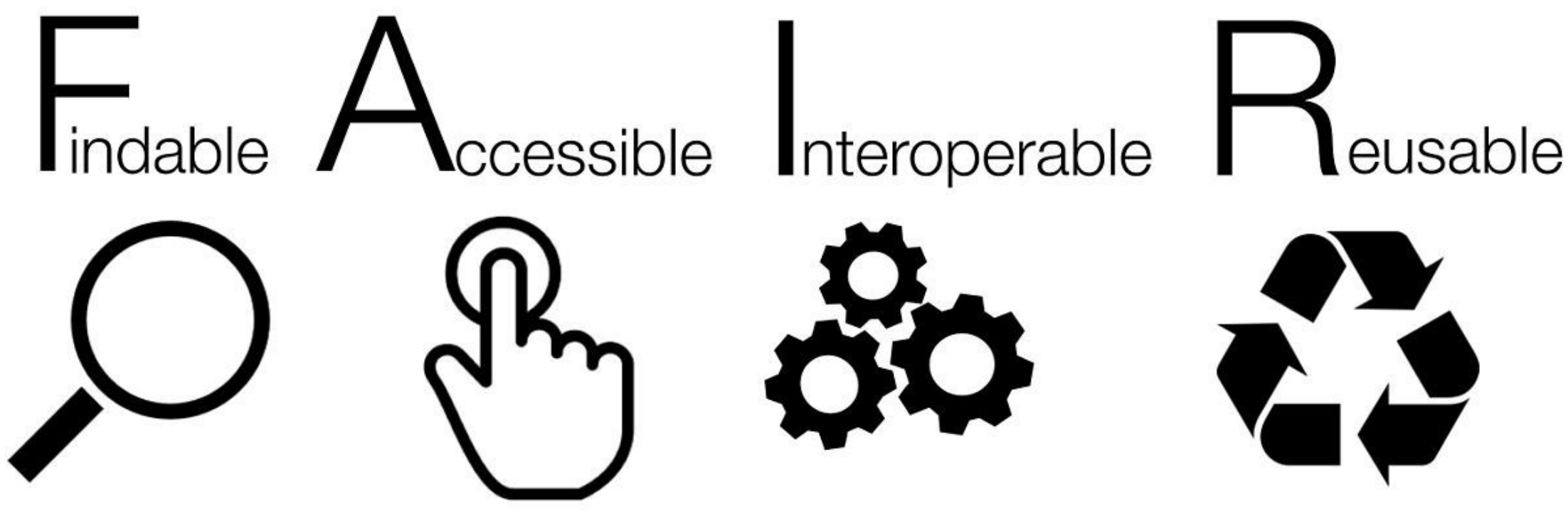

\section{Metadata}

ISO 19115-2

Dublin Core

GCMD DIF

Frictionlessdata Data Package
Vocabularies

Schema.org

Dublin Core

Dataset Catalog (DCAT)

GeoSPARQL

PROV-O

Ocean Data Ontology

\section{Services}

Google Dataset Search

Linked Data

Geospatial Search

ERDDAP

Data Packaging

Custom APIs
BCO-DMO Data@Google lod.bco-dmo.org/browse/ bco-dmo.org/search/dataset erddap.bco-dmo.org frictionlessdata.io C-DEBI, NSIDC, R2R, LTER

- Original article describing FAIR data principles: Wilkinson, M.D., et al. (2016) The FAIR Guiding Principles for scientific data management and stewardship. Scientific Data, 3 , 160018, doi:10.1038/sdata.2016.18

- Article: Stall, S., et al. (2018), Advancing FAIR data in Earth, space, and environmental science, Eos, 99, doi:10.1029/2018EO109301 


\section{Submission Details}

$>$ Engage when you receive your funding

$>$ Extensive metadata model

$>$ No restrictions on data size

$>$ Accepts model output (and inputs + code)

$>$ Personalized support from a Data Manager

Danie, Adam, Shannon, Matt, Amber, Tina, Karen \& Nancy
$>$ Training available

$>$ Data Licenses (CC BY 4.0)

$>$ DOIs

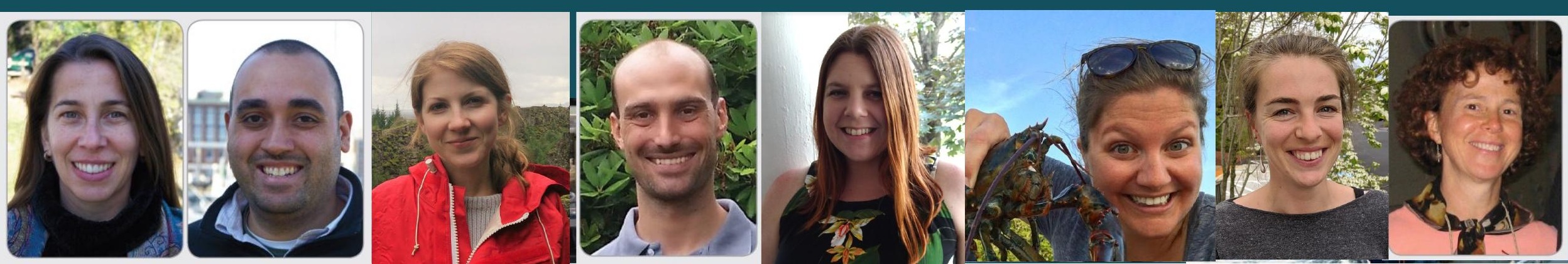




\section{Learn more at the BCO-DMO booth!}

\#321 